\title{
Interspecific Hybridization of Meiotic Parthenogenetic Meloidogyne chitwoodi and M. fallax
}

\author{
J. G. van der Beek and G. Karssen
}

First author: Research Institute for Plant Protection (IPO-DLO), P.O. Box 9060, 6700 GW Wageningen, the Netherlands; second author: Plant Protection Service (PD), P.O. Box 9102, 6700 HC Wageningen, the Netherlands.

Accepted for publication 16 June 1997.

\begin{abstract}
Van der Beek, J. G., and Karssen, G. 1997. Interspecific hybridization of meiotic parthenogenetic Meloidogyne chitwoodi and M. fallax. Phytopathology 87:1061-1066.

Hybridization between two meiotic parthenogenetic species of rootknot nematodes, Meloidogyne chitwoodi and M. fallax, was investigated in two different crossing experiments on tomato plants grown in sand. The first experiment was a controlled cross between the two species. The second experiment was a bulk mating in a 1:1 mixture of two isolates. The haploid chromosome number of the parental isolates was $n=18$. Successful interspecific hybridization was obtained, and the resulting hybrids produced egg masses. In eggs, cell division was observed, but most of them were without clear differentiation and consequently were

were present in equal numbers, and $10 \%$ of all females were nonviable hybrids. Similar ratios of parental-type and hybrid females were detected in roots of test plants grown in soil from a field sample that contained a mixture of $M$. chitwoodi and M. fallax populations. In the controlled cross experiment, isozyme electrophoresis of malate dehydrogenase was applied to distinguish the two species and their hybrids. In the bulk mating experiment, malate dehydrogenase, esterase, and glucose 6-phosphate dehydrogenase were used as markers, two by two simultaneously on the same individual females, providing conclusive evidence for the occurrence of hybrids. This is the first report on interspecific hybridization in Meloidogyne. The possible role of interspecific hybridization in species differentiation and interspecific exchange of genetic material within Meloidogyne is discussed.
\end{abstract} sterile. Hatched $\mathrm{F}_{2}$ juveniles were small in number, not viable, and showed morphological distortions. In the progeny of the isolate mixture of the bulk mating experiment, parental-type females of the two isolates
Additional keywords: genetic variation, speciation.
Reproduction in the root-knot nematodes Meloidogyne chitwoodi Golden et al. and $M$. fallax Karssen $(11,16)$ is characterized by facultative meiotic parthenogenesis (30), i.e., amphimixis and meiotic parthenogenesis can occur in these species. Amphimixis may result when insemination by males occurs. Until recently, $M$. chitwoodi and M. fallax were considered one species, and M. fallax has been referred to as M. chitwoodi type Baexem $(34,37)$. During the past few years, significant differences between the two species have been reported. Van Meggelen et al. (34) mentioned enzymatic differences between M. chitwoodi and M. chitwoodi type Baexem and suggested race status. Karssen (17) briefly described morphological and biochemical differences, and Zijlstra et al. (37) showed differences in internal transcribed spacer (ITS) regions of ribosomal DNA. Finally, additional morphological differences between the two species and specific hosts were described (16). These species are distinct from each other in morphological, morphometrical, biochemical, and biological characters, but their reproductive isolation has not been demonstrated.

In a few other nematode species, interspecific hybridization has been proven: Heterodera spp. $(9,21,26,36)$, Globodera spp. (10, 21,22,28), and Pratylenchus spp. (24). The objective of this study was to investigate reproductive isolation in M. chitwoodi and M. fallax, the results of which might explain the role of interspecific hybridization in genetic divergence in the genus and speciation $(19,20)$. To examine reproductive isolation, hybridization between M. chitwoodi and M. fallax was studied in a strictly controlled experiment and a bulk mating experiment.

Corresponding author: J. G. van der Beek

E-mail address: j.g.vanderbeek@IPO.DLO.NL

Publication no. P-1997-0827-01R

(C) 1997 The American Phytopathological Society

\section{MATERIALS AND METHODS}

Nematode history and identification. Crossing experiments were conducted with isolates $\mathrm{Ca}$ and $\mathrm{Ci}$ of $M$. chitwoodi and isolate $\mathrm{Fa}$ of $M$. fallax, all three originated from geographically different sites in the Netherlands (Table 1). M. javanica (Treub.) Chitwood, isolate $\mathrm{Ja}$, which originated from China, was used as a reference phenotype in isozyme electrophoresis (Table 1). The isolates were obtained from separated field populations maintained in a greenhouse on tomato (Lycopersicon esculentum Mill. 'Moneymaker') and regularly checked for species identity. These isolates were identified as true species by a combination of morphological characteristics $(14,16)$, isozyme patterns (8), and DNA patterns of ITS regions (37) (Table 1).

Controlled crossing experiment. Reciprocal crosses were made between M. chitwoodi and M. fallax. Isolates $\mathrm{Ca}$ and $\mathrm{Fa}$ were allowed to hybridize, using a modification of the procedure used by Triantaphyllou (32). Two-week-old tomato seedlings, grown on silversand enriched with 3\% slow release fertilizer Osmocote Plus (15 $+11+13[\mathrm{~N}+\mathrm{P}+\mathrm{K}]+2 \mathrm{MgO}+$ micros [Scotts Europe BV]) in open-ended transparent plastic tubes $(96 \mathrm{ml})$, were inoculated with prehatched second-stage juveniles (J2). To obtain the paternal line, plants were inoculated on 23 June 1995 with isolates $\mathrm{Ca}$ and $\mathrm{Fa}$ to a final density of $\sim 45 \mathrm{~J} 2$ per $\mathrm{ml}$ of soil. This high density enhanced the number of $\mathrm{J} 2$ that developed into males, because the gender ratio in Meloidogyne is strongly influenced by nematode density $(6,29)$. One week later, roots of additional seedlings were inoculated with nematode suspensions of the same two isolates, at a rate of $\sim 2.5 \mathrm{~J} 2$ per $\mathrm{ml}$ of soil, to generate the maternal line. This low density caused most of the J2 to develop into females. The plants were placed in a growth cabinet at $20^{\circ} \mathrm{C}, 70 \%$ relative humidity $(\mathrm{RH})$, and $16 / 8 \mathrm{~h}$ of light/dark. Fourteen days after inoculation, roots from the paternal line were placed on sieves in a mist cabinet at $100 \% \mathrm{RH}$, mist temperature of $28^{\circ} \mathrm{C}$, and ambient temperature of $18^{\circ} \mathrm{C}$. This high 
humidity caused a thin water film to run from the roots, by which males left the roots and accumulated at the bottom of the receptacle. After 10 days, males were collected and added to the plants containing the maternal line. This procedure was repeated eight times during a period of 17 days, each time adding 10 to 50 males.

Females and their corresponding egg masses were collected separately from roots on 24 August 1995. To examine whether females were inseminated, spermatothecae and oocytes of these females were checked for the presence of sperm by making smear preparations (31) that were stained with Hoechst 33258 (procedure used by Albertson et al. [1], with modifications). In inseminated females, male gametes accumulate in the spermatotheca, which is a specialized structure in the female gonad. A male gamete fertilizes the oocyte that enters the spermatotheca, normally during metaphase I. To detect the required interspecific first generation, hatched J2 of egg masses of inseminated females were inoculated individually on 8 September 1995 on Solanum tuberosum L. 'Eigenheimer' root tips grown in petri dishes on water agar, with only one J2 per petri dish. These petri dishes were incubated for 7 weeks under dark conditions in a growth cabinet at $20^{\circ} \mathrm{C}$. Females with

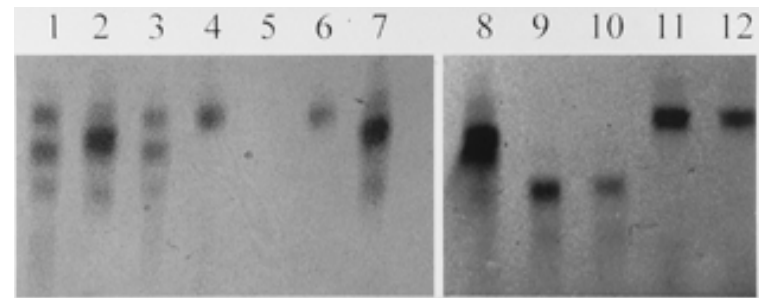

Fig. 1. Malate dehydrogenase zymograms showing representative samples of parental and cross-hybrid phenotypes of individual females of controlled crosses between Meloidogyne chitwoodi $\mathrm{Ca}$ and $M$. fallax Fa. Lanes 1 and 3, hybrid phenotypes; lanes 4 and 6 , maternal $M$. chitwoodi Ca phenotypes; lane 5, absent or null; lanes 9 and 10, parental reference phenotypes for $M$. fallax $\mathrm{Fa}$; lanes 11 and 12, parental reference phenotypes for $M$. chitwoodi Ca. Lanes 2, 7, and 8, M. javanica Ja used as a reference phenotype. their corresponding egg masses were collected separately and used for electrophoresis and a hatching test, respectively.

Bulk mating experiment. A second experiment with isolates $\mathrm{Ci}$ and $\mathrm{Fa}$ was conducted from June to October 1995 to test the occurrence of spontaneous mating between $M$. chitwoodi and $M$. fallax. The M. chitwoodi isolate $\mathrm{Ci}$ was different from the isolate used in the controlled cross to investigate whether the possibility of hybridization with different isolates does exist. Two-week-old tomato seedlings were transplanted to 3.2-liter clay pots containing sterilized sandy soil, $33 \%$ (vol/vol) water-retaining granules (Hydrocorn; Jongkind Hydro bv, Aalsmeer, the Netherlands), and $3 \%$ (wt/vol) Osmocote. One- to two-day-old prehatched J2 were used as inoculum. Three weeks after sowing four tomato seedlings were inoculated, each with $\sim 3.3 \mathrm{~J} 2$ per $\mathrm{ml}$ of soil, comprising a 1:1 mixture of $\mathrm{Ci}$ and $\mathrm{Fa}$ juveniles, and placed in a greenhouse at $20^{\circ} \mathrm{C}$ ( 18 to $\left.24^{\circ} \mathrm{C}\right)$. To complete approximately two nematode generations, the infected plants were maintained for 14 weeks, based on known population development (25). The gender ratio was expected to develop toward a female majority during the first generation, with only a low frequency for possible interspecific hybridization. To detect hybrids and test the viability of the progeny of these hybrids, the experiment was terminated after about the second generation, which approximated the first hybrid generation. From each tomato plant, 40 females with their corresponding egg masses were collected separately and used for electrophoresis and a hatching test, respectively.

Chromosome counting. The chromosomes of the parental lines were examined. Approximately 25 young egg-producing females were collected from infected roots of each parental line, and smear preparations were made (31) and stained with Hoechst 33258. The chromosomes were counted in oocytes during early metaphase I and just after the first maturation division.

Hybrid detection and $F_{1}$ progeny viability. Collected females and corresponding egg masses from both experiments were kept at -80 and $20^{\circ} \mathrm{C}$, respectively. Due to the facultative parthenogenetic nature of the isolates, females can produce parthenogenetic or sex-

TABLE 1. Specific characteristics of the Meloidogyne spp. isolates used in this study

\begin{tabular}{lcclccc}
\hline Species & Isolate & Sampling year & Original code & EST & MDH $^{\mathrm{c}}$ & HinfI $^{\mathrm{d}}$ \\
\hline M. chitwoodi & Ca & 1991 & C3022 (PD) & S1 & N1a & 440,270 \\
M. chitwoodi & Ci & 1991 & C5273-C (PD) & S1 & N1a & 440,270 \\
M. fallax & Fa & 1992 & CHB (PAGV) & F3 & N1b & 430,270 \\
M. javanica & Ja & 1990 & C3059 (PD) & J3 & N1 & 460 \\
\hline
\end{tabular}

a PD = Plant Protection Service, Wageningen, the Netherlands. PAGV = Research Station for Arable Farming and Field Production of Vegetables, Lelystad, the Netherlands.

${ }^{\mathrm{b}}$ Isozyme phenotype for esterase (6,15). F3 = Unique $E S T$ phenotype for $M$. fallax .

${ }^{\mathrm{c}}$ Isozyme phenotype for malate dehydrogenase (6). N1b = Unique $M D H$ phenotype for $M$. fallax.

d Discriminating restriction products (in base pairs) when restricted with HinfI of the 760-bp polymerase chain reaction-amplified internal transcribed spacer (ITS) regions (33).

${ }^{\text {e }}$ Discriminating restriction product (in base pairs) when restricted with $R s a \mathrm{I}$ of the 760-bp polymerase chain reaction-amplified ITS regions (33).
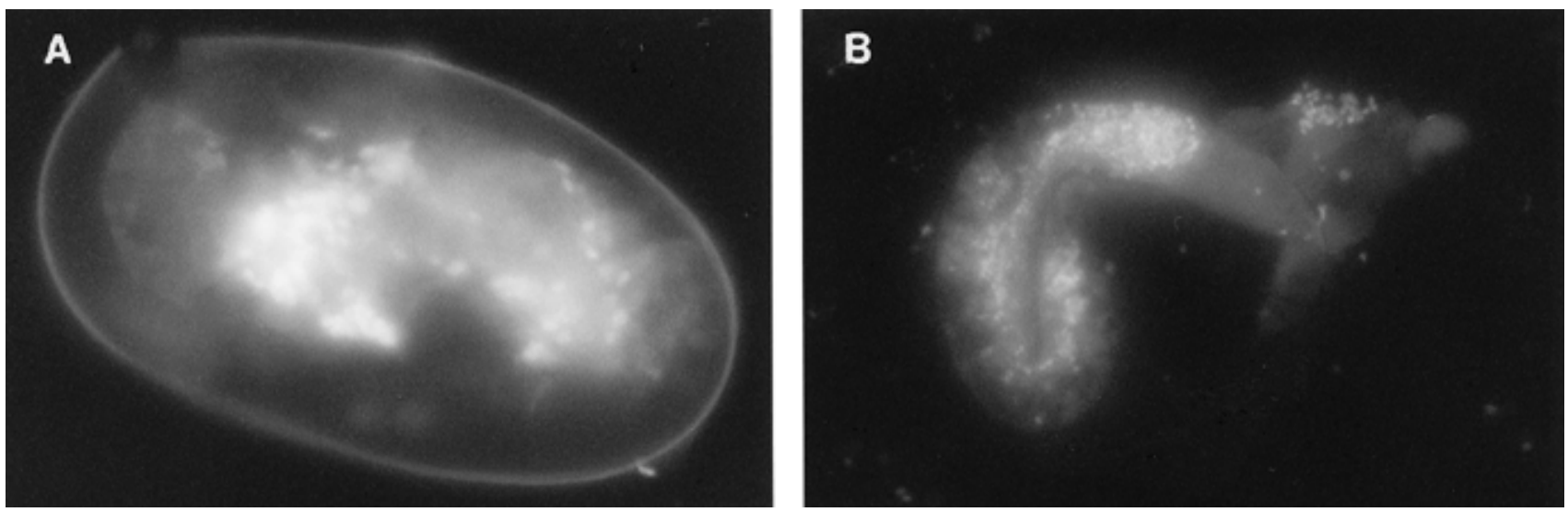

Fig. 2. Eggs of Meloidogyne chitwoodi $\times$ M. fallax hybrids at $\sim 1$ month after hatching, stained with Hoechst 33258 . A, Undifferentiated mass of nuclei. B, Malformed juvenile. 
ual offspring. The latter may be the result of insemination of females by either males of the maternal or paternal line. Moreover, more than one male may fertilize one female. Consequently, parthenogenetically and sexually produced eggs may occur in an egg mass of an inseminated female, and the progeny of a crossing may contain both parthenogenetic and sexual offspring. In such a progeny, true hybrid females can be distinguished from a cross with a male from the maternal line and a parthenogenetic offspring by a differentiating marker between the two species. Three independent isozyme markers were used: malate dehydrogenase ( $M D H$; EC 1.1.1.37), esterase (EST; EC 3.1.1.1), and glucose 6-phosphate dehydrogenase (G6PD; EC number 1.1.1.49). $M D H$ was applied in the controlled crossing experiment, and $M D H, E S T$, and G6PD were applied in the bulk mating experiment. To provide conclusive evidence for hybri- dization, individual females were subjected to isozyme electrophoreses of $M D H$ and EST, and other females were subjected to electrophoresis of $M D H$ and $G 6 P D$, resulting in two phenotypes for one female with independent isozyme markers. Isozyme electrophoresis and staining were carried out on microgels according to Karssen et al. (18). Egg masses were collected individually in microvials with $10 \mathrm{ml}$ of water and placed in a $20^{\circ} \mathrm{C}$ incubator to hatch. During a period of 8 weeks, hatching was checked weekly.

\section{RESULTS}

Hybrids in the controlled crossing experiment. The haploid chromosome number of both parental isolates in the controlled crossing experiment, $\mathrm{Ca}$ and $\mathrm{Fa}$, was the same $(n=18)$. No $\mathrm{F}_{1}$
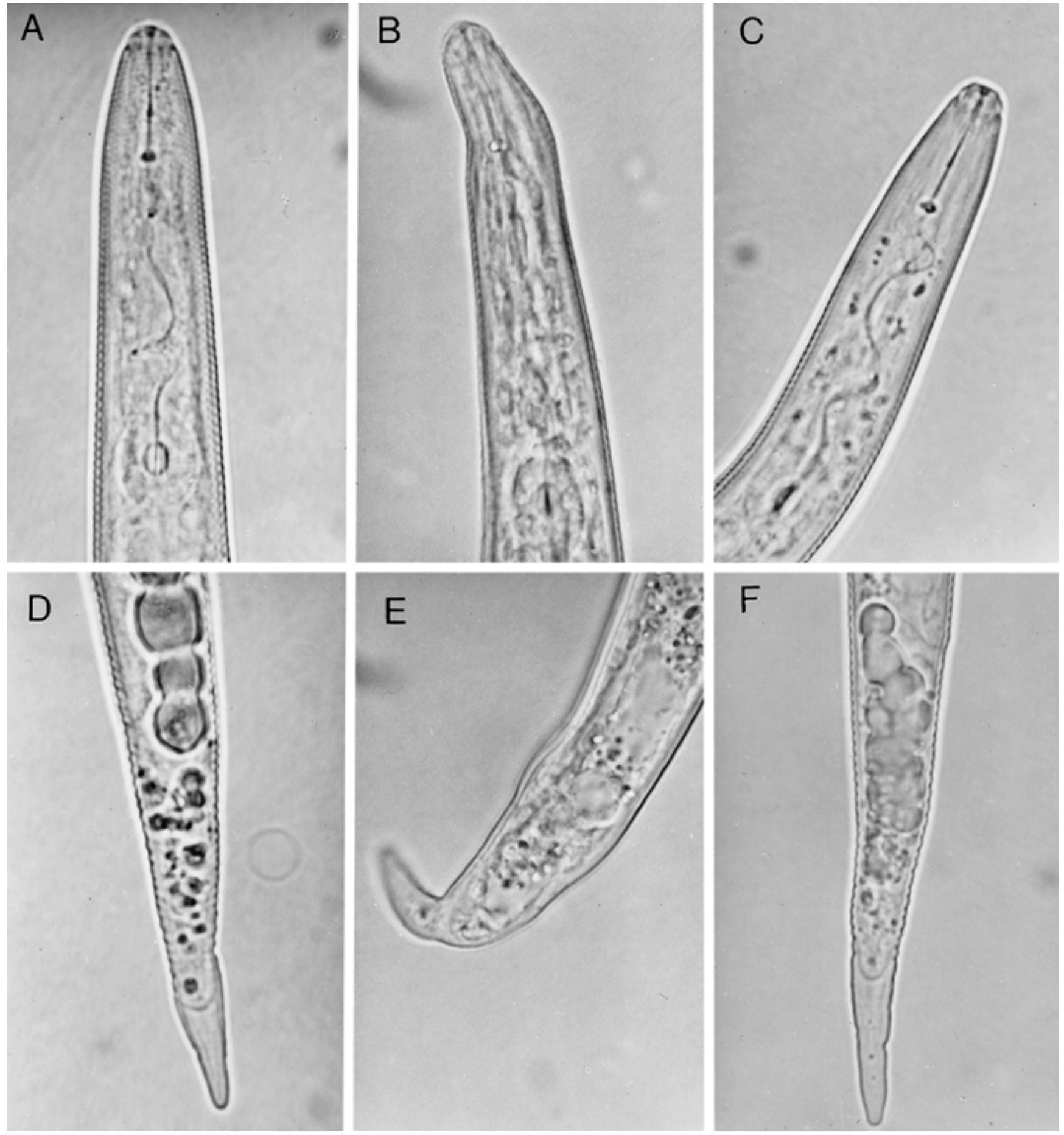

Fig. 3. Characteristic head and tail structures of a juvenile of a Meloidogyne chitwoodi $\times$ M. fallax hybrid compared with its parents. A and D, M. chitwoodi. B and E, Progeny of hybrid. C and F, M. fallax. 
hybrids were obtained from the cross of $M$. fallax and $M$. chitwoodi, with $M$. fallax as the female parent, because the $M$. chitwoodi male parent did not produce sufficient males for the experiment. This has been noticed often during maintenance of $M$. chitwoodi isolates. $\mathrm{F}_{1}$ hybrids were obtained from the cross of $M$. chitwoodi and $M$. fallax, with $M$. chitwoodi as the female parent. Seven females were collected from two plants, of which one female contained sperm, which was detected in the spermatotheca and oocytes. Originating from the egg mass of this inseminated female, 60 hatched $\mathrm{J} 2$ were inoculated separately on root tips, resulting in 21 females with egg masses. Electrophoresis for $M D H$ resulted in two isozyme patterns: eight females exhibited only the maternal (M. chitwoodi) isozyme band (Fig. 1, lanes 4 and 6), and four females exhibited patterns, with three regularly spaced bands corresponding to the two parental bands and an intermediate band (Fig. 1, lanes 1 and 3). For nine females, no clear bands were found (Fig. 1, lane 5), probably due to loss of female material during preparation of the electrophoresis samples. Although a successful cross was obtained, none of the four hybrid females with clear intermediate bands produced normal fertile offspring, in contrast to all of the females showing maternal isozyme patterns. Of the nine females without clear isozyme bands, two produced offspring, and seven did not. Assuming that these 7 were also true hybrids, a total of 11 interspecific hybrid females and 10 parthenogenetic progeny was obtained. Egg masses of hybrid females contained up to 100 eggs each. Most eggs were able to develop into a multicellular undifferentiated structure, and in some eggs, a juvenile was visible (Fig. 2). Some juveniles were even able to hatch at $20^{\circ} \mathrm{C}$ but were distorted, showing deviating head and tail shapes (Fig. 3), and died shortly after hatching.

Hybrids in the bulk mating experiment. The haploid chromosome number of isolate $\mathrm{Ci}$, used as one parent in the bulk mating experiment of $M$. chitwood $i$ with $M$. fallax, was $n=18$, like the isolates $\mathrm{Ca}$ and $\mathrm{Fa}$. As a result of this crossing experiment, 77 and 67 parental-type females of $M$. chitwoodi and M. fallax, respectively, were obtained and detected, with $M D H$ as marker, 14 weeks after inoculation (Table 2; Fig. 4). Additionally, $16 \mathrm{~F}_{1}$ hybrid females were detected, which was $10 \%$ of the total number of examined females. None of the hybrid females produced viable offspring, whereas all parental-type females produced normal progeny. Parental-type and hybrid females showed corresponding parental and hybrid patterns for the two isozymes $M D H$ and EST (Fig. 5), as well as for $M D H$ and $G 6 P D$ (Fig. 6) on the zymograms, comparable to $M D H$ (Fig. 1) in the controlled crossing experiment.

\section{DISCUSSION}

This is the first report of successful interspecific crossing within the genus Meloidogyne, demonstrating hybridization between $M$. chitwoodi and M. fallax. Ample evidence for interspecific hybridization was obtained in this study from controlled and bulk crosses. Triantaphyllou successfully hybridized $M$. hapla isolates ( $n=15$ [\%] and 17 [^] chromosomes) (A. C. Triantaphyllou, personal communication). Hybrid progeny had $n=16$ or 17 chro-

TABLE 2. Number of parental-type and hybrid females in the bulk mating experiment of Meloidogyne chitwoodi isolate $\mathrm{Ci}$ and $M$. fallax isolate $\mathrm{Fa}$

\begin{tabular}{llccc}
\hline Tomato plant & $\mathrm{Ci}^{\mathrm{a}}$ & $\mathrm{Fa}^{\mathrm{b}}$ & Hybrid $^{\mathrm{c}}$ & Total $q$ + \\
\hline 1 & 16 & 20 & 4 & 40 \\
2 & 24 & 12 & 4 & 40 \\
3 & 17 & 18 & 5 & 40 \\
4 & 20 & 17 & 3 & 40 \\
Total & 77 & 67 & 16 & 160 \\
\hline
\end{tabular}

a Parental-type females possessing the N1a band ( $M D H$ phenotype of $\mathrm{Ci}$ ).

b Parental-type females possessing the N1b band ( $M D H$ phenotype of Fa).

c Hybrid-type females possessing the N1a and N1b bands and an intermediate band. mosomes. Pairing of homologous chromosomes was not perfect, because loops were observed in some bivalents during diakinesis; nevertheless, hybrid progeny were propagated successfully for many generations. Attempts to hybridize M. chitwoodi with M. hapla, however, were unsuccessful, as detected with chromosome and biochemical markers (A. C. Triantaphyllou, personal communication; 33). In this study, the isolates used for crosses appeared to possess identical chromosome numbers. No data are available on crosses between $M$. chitwoodi and M. fallax with different chromosome numbers. Differences in chromosome number between parental isolates are expected to interfere with hybridization.

The current study supports the separate species status of $M$. chitwoodi and $M$. fallax, based on the nonviable progenies of their interspecific hybrids. Triantaphyllou (30) stated that “...conceptually they (facultatively meiotic parthenogenetic species) can be considered as true biological species if they are really reproductively isolated from each other. However, tests for demonstrating reproductive isolation are technically very difficult because of the facultative nature of parthenogenetic reproduction and the lack of genetic markers that would verify intercrossing." By demonstrating reproductive isolation between the two meiotic parthenogenetic species in this study, it is now evident that they are true biological species.

Isozyme electrophoresis was a convenient tool for detecting interspecific hybridization, using isozymes as genetic markers, as
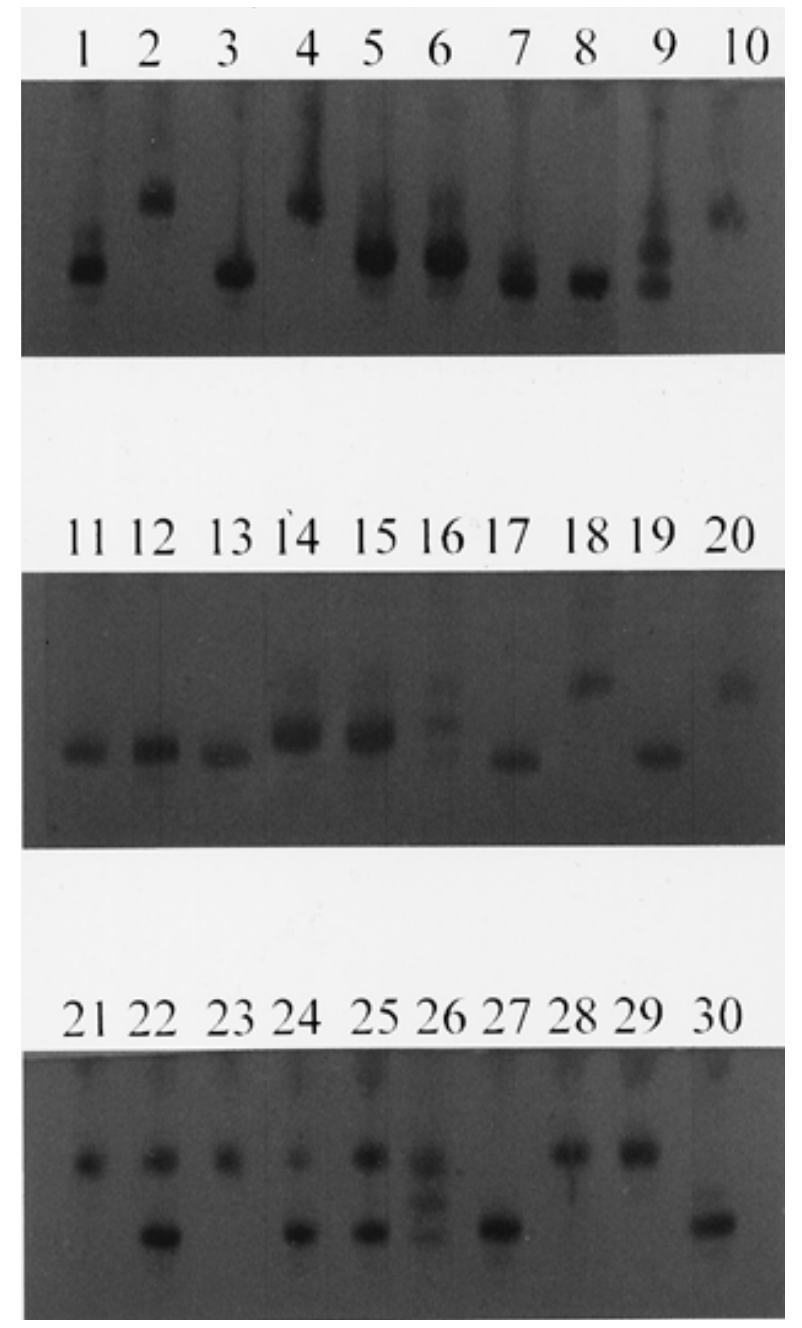

Fig. 4. Malate dehydrogenase zymograms showing representative samples of parental and cross-hybrid phenotypes of the bulk mating experiment between $\mathrm{Mel}$ oidogyne chitwoodi $(\mathrm{Ci})$ and $M$. fallax $(\mathrm{Fa})$ and phenotypes of mixtures of females of the two species. Lanes 2, 4, 10, 18, 20, 21, 23, 28, and 29, phenotypes of $M$. chitwoodi. Lanes 1, 3, 7, 8, 11, 12, 13, 17, 19, 27, and 30, phenotypes of M. fallax. Lanes 9, 16 and 26, hybrids. Lanes 22, 24, and 25, species mixtures. Lanes 5, 6, 14, and 15, M. javanica (Ja) used as a reference phenotype. 
has been shown previously in certain insects, for example waterflea (Daphnia spp.) (35), stick-insects (Bacillus spp.) (3), and planthopper (Muellerianella spp.) (2). Van Meggelen et al. (34) stated that $M$. chitwoodi and $M$. fallax have different $M D H$ phenotypes. The hybrid was expected to possess three distinct bands in a zymogram: both parental bands and an intermediate band, because $M D H$ consists of two polypeptide chains, and a heterodimer is expected to be formed in a heterozygote (23), in contrast to a mixture of species that show only the two parental bands (Fig. 4). The three $M D H$ patterns found in this study correspond to genotypes for the two parental species and their hybrid, confirming the codominant nature of locus $M D H . M D H$ is a stable marker for distinguishing species of Meloidogyne, showing identical isozyme patterns within one species, with a few exceptions in $M$. arenaria, $M$. incognita, and $M$. javanica (7). $M D H$ genotypes of $\sim 100 \mathrm{fe}-$ males of $M$. chitwoodi and 60 of $M$. fallax, representing various isolates, were characteristic for species, showing identical phenotypes, as described in this paper (J. G. van der Beek and G. Karssen, unpublished data). The genotypes of the hybrid females in this study, which exhibit hybrid patterns for the independent markers $M D H$ and $E S T$ and for $M D H$ and G6PD in the same individuals, provide conclusive evidence for hybridization between $M$. chitwoodi and $M$. fallax (Figs. 5 and 6). G6PD, which distinguishes $M$. chitwoodi from $M$. fallax (4), is also a heterodimer, and in the hybrid, the middle band

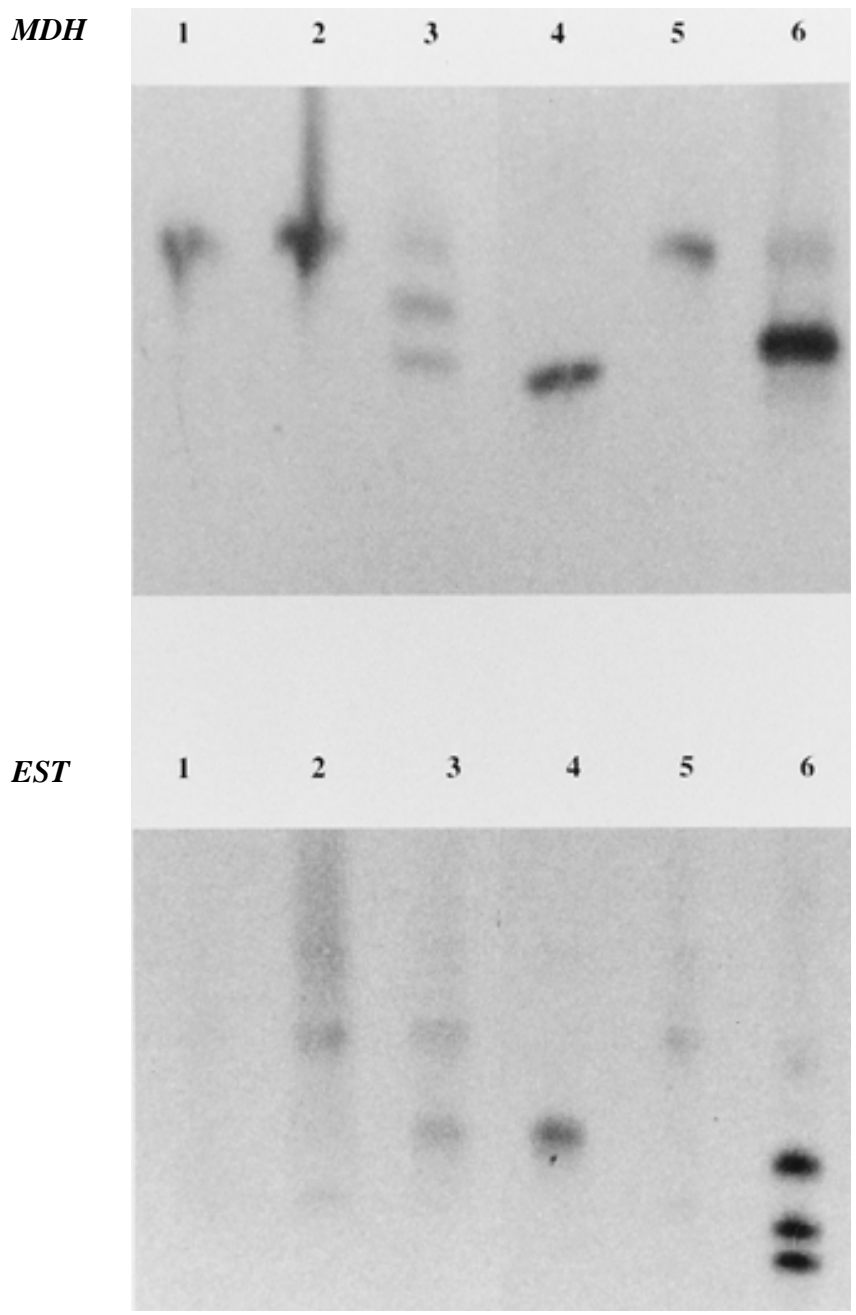

Fig. 5. Zymograms of malate dehydrogenase $(M D H)$ and esterase $(E S T)$, showing representative samples of parental and cross-hybrid phenotypes for both isozymes on the same females used in the bulk mating between $\mathrm{Mel}$ oidogyne chitwoodi $(\mathrm{Ci})$ and $M$. fallax $(\mathrm{Fa})$. Lane 4, phenotypes of $M$. chitwoodi. Lanes 1, 2, and 5, phenotypes of $M$. fallax. Lane 3, phenotypes of the hybrid. Lane 6, M. javanica (Ja) used as a reference. $M D H$ and EST phenotypes for $M$. fallax are designated N1b and F3, respectively. is predominantly visible. EST is a monomer, and its phenotype for M. fallax has been described as a "null" (16). However, when stained for $90 \mathrm{~min}$, a faint three-band pattern appears that has not been described yet and is denoted as F3 (Fig. 5, lanes 2 and 5). The hybrid EST pattern shows a strong band corresponding to the $M$. chitwoodi S1 phenotype and the slower band of the M. fallax F3 phenotype (Fig. 5). The $M D H$ phenotype of $M$. fallax, although described previously (16), is designated as N1b.

Not all females were expected to be fertilized by males. Mating in parthenogenetic Meloidogyne spp. occurs by chance (27). However, even if females were inseminated, it could have been entirely or partly by males of the maternal isolate. Additionally, the egg mass from an inseminated female was expected to contain an unknown portion of unfertilized, parthenogenetic eggs. This explains the occurrence of maternal-type females in the progeny of an inseminated female in the controlled crossing experiment. Furthermore, it explains why a Mendelian segregation is not expected in crossings between meiotic parthenogenetic Meloidogyne spp.

Interestingly, the two species used in this study are either allopatric or sympatric; both are prevalent in the southeastern Netherlands. Because of their similarity in ecological niche, in total protein patterns (J. G. van der Beek, unpublished data), and in sharing many host plants, including monocotyledons, it could be that both species share a common ancestry. In our experiments

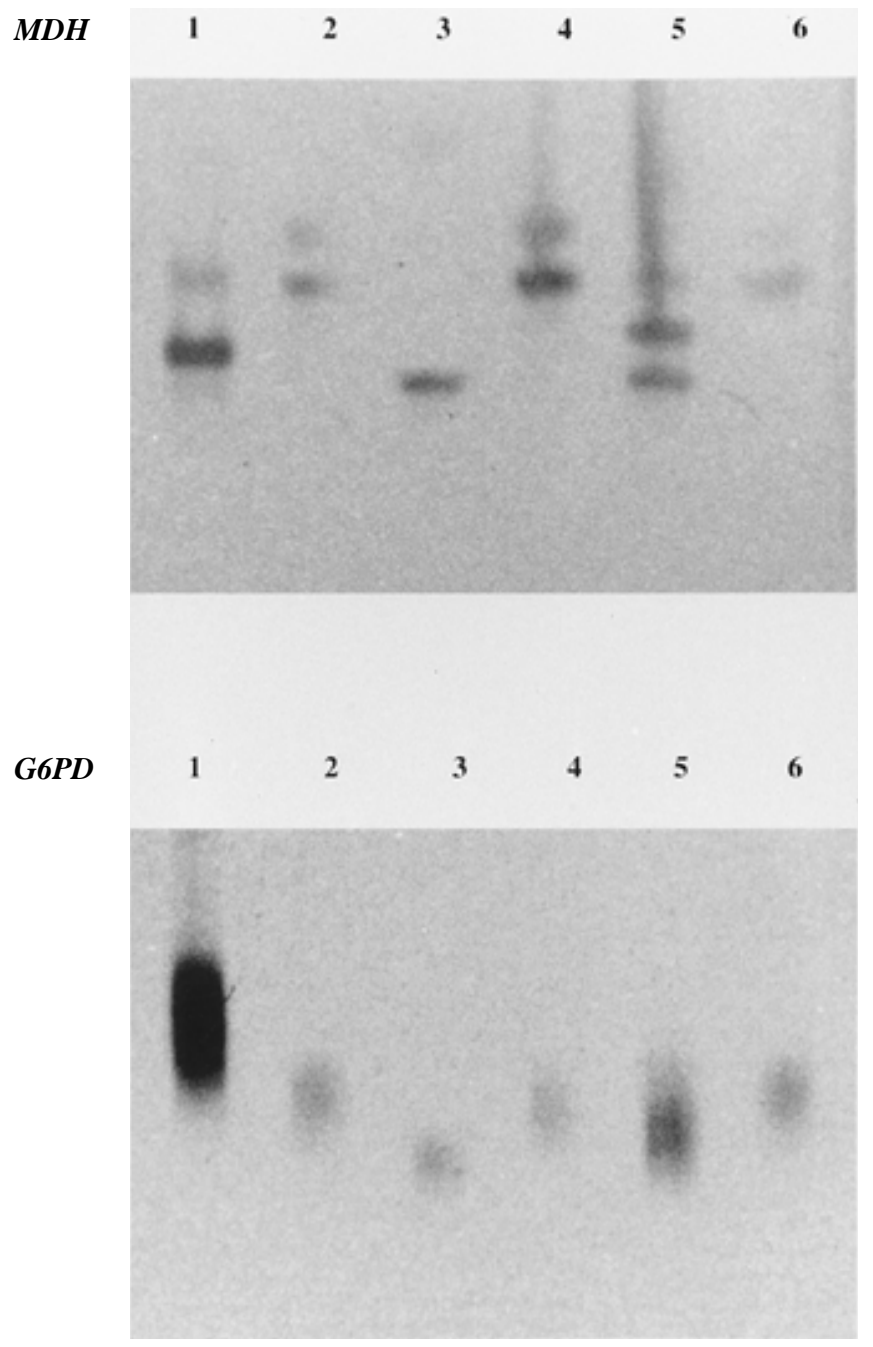

Fig. 6. Zymograms of malate dehydrogenase $(M D H)$ and glucose 6-phosphate dehydrogenase $(G 6 P D)$ showing representative samples of parental and cross-hybrid phenotypes for both isozymes on the same females used in the bulk mating between Meloidogyne chitwoodi $(\mathrm{Ci})$ and $M$. fallax (Fa). Lane 3, phenotypes of $M$. chitwoodi. Lanes 2, 4, and 6, phenotypes of $M$. fallax. Lane 5 , phenotypes of the hybrid. Lane $1, M$. javanica (Ja) used as a reference. 
with mixtures of isolates, hybrids between $M$. chitwoodi and $M$. fallax occurred regularly. Although deformed and nonviable, a very few juveniles were found in the offspring of these hybrids. Speculatively, hybrids may occasionally produce fertile progeny, because they are able to backcross to one of the parents, as is shown in interspecific hybrids in Daphnia $(12,35)$. For Meloidogyne, this would signify that exchange of DNA could be possible, including genes for avirulence/virulence. If so, successful breeding for durable crop plant resistance should include testing of isolates of all potentially aggressive species that can interbreed.

In addition to broadening the genetic base of a species by backcrosses of the hybrid to a parental population, hybridization also may play an important role in speciation in certain organisms (19). Two steps are required for a species to arise from interspecific hybridization (3): interspecific hybridization and changes in the maturation divisions that allow transmission of the hybrid genome to the next generation. The latter changes are undoubtedly rare. Dalmasso and Bergé (5) speculated that $M$. javanica, a polyploid, mitotic, parthenogenetically reproducing species, may have arisen in this way, because of its high level of heterozygosity for some isozymes. One of its parents could have been $M$. hapla, based on similarity in nucleotide sequences between $M$. javanica and M. hapla (13).

In addition to the results described in this paper, more evidence for hybridization between $M$. chitwoodi and M. fallax was derived from observations on lettuce roots in which parental and hybrid females were detected. Four of forty females showed hybrid $M D H$ phenotypes similar to those presented in Figures 1 and 4. These lettuce plants were grown in soil that originated from a field in which an almost equal mixture of populations of the two species was detected.

Mixtures of Meloidogyne species are found more frequently under natural conditions. In these situations, hybridization may be feasible, and under environmentally favorable conditions for host plant rotation, intensity of land use, and soil characteristics, it is likely that new forms of Meloidogyne may appear.

\section{ACKNOWLEDGMENTS}

We thank L. M. Poleij and T. van Hoenselaar for their technical assistance. We also thank P. van Halteren, R. F. Hoekstra, P. W. Th. Maas, C. H. van Silfhout, and C. Zijlstra for critical reading of the manuscript.

\section{LITERATURE CITED}

1. Albertson, D. G., Sulston, J. E., and White, J. G. 1978. Cell cycling and DNA replication in a mutant blocked in cell division in the nematode Caenorhabditis elegans. Dev. Biol. 63:193-221.

2. Booij, C. J. H. 1982. Biosystematics of the Muellerianella complex (Homopetra, Dephacidae): Host plants, habitats and phenology. Ecol. Entomol. 7:9-18.

3. Bullini, L., and Nascetti, G. 1989. Speciation by hybridization in insects. Pages 317-339 in: Electrophoretic Studies on Agricultural Pests. Systematic Association Special Volume 39. H. D. Loxsale and J. Den Hollander, eds. Clarendon Press, Oxford.

4. Daher, S., Gillet, S., Mugniéry, D., and Marzin, H. 1996. Discovery in France and characteristics of the Dutch variant of Meloidogyne chitwoodi. Page 188 in: Proc. 3rd Int. Nematol. Congr. INRA, ORSTOM, CIRAD, Gosier, Guadeloupe, French West Indies.

5. Dalmasso, A., and Bergé, J. B. 1983. Enzyme polymorphism and the concept of parthenogenetic species, exemplified by Meloidogyne. Pages 187-196 in: Concepts in Nematode Systematics. Systematics Association Special Volume 22. A. R. Stone, H. M. Platt, and L. F. Khalil, eds. Academic Press, London.

6. Davide, R. G., and Triantaphyllou, A. C. 1967. Influence of environment on development and sex differentiation of root-knot nematodes. Nematologica 13:102-110.

7. Esbenshade, P. R., and Triantaphyllou, A. C. 1985. Use of isozyme phenotypes for identification of Meloidogyne species. J. Nematol. 17:6-20.

8. Esbenshade, P. R., and Triantaphyllou, A. C. 1990. Isozyme phenotypes for the identification of Meloidogyne species. J. Nematol. 22:10-15.

9. Fox, J. A. 1967. Reproductive isolation in the genus Heterodera. Nema- tologica 13:143-144.

10. Franco, J., and Evans, K. 1978. Mating of British and Peruvian populations of potato cyst nematodes Globodera spp. Nematropica 8:5-9.

11. Golden, A. M., O'Bannon, J. H., Santo, G. S., and Finley, A. M. 1980. Description and SEM observations of Meloidogyne chitwoodi n. sp. (Meloidogynidae), a root-knot nematode on potato in the Pacific Northwest. J. Nematol. 12:319-327.

12. Hebert, P. D. N. 1985. Interspecific hybridization between cyclic parthenogenesis. Evolution 39:216-220.

13. Hyman, B. C., and Powers, T. O. 1991. Integration of molecular data with systematics of plant parasitic nematodes. Annu. Rev. Phytopathol. 29:89-107.

14. Jepson, S. B. 1987. Identification of the root-knot nematodes (Meloidogyne species). Commonwealth Agricultural Bureaux, Farnham Royal, England.

15. Karssen, G. 1995. Morphological and biochemical differentiation in Meloidogyne chitwoodi populations in the Netherlands. Nematologica 41:314-315.

16. Karssen, G. 1996. Description of Meloidogyne fallax $\mathrm{n}$. sp. (Nematoda: Heteroderidae), a root-knot nematode from the Netherlands. Fundam. Appl. Nematol. 19:593-599.

17. Karssen, G. 1995. Morphological and biochemical differentiation in Meloidogyne chitwoodi populations in the Netherlands. (Abstr.) Nematologica 41:314-315.

18. Karssen, G., van Hoenselaar, T., Verkerk-Bakker, B., and Janssen, R. 1995. Species identification of cyst and root-knot nematodes from potato by electrophoresis of individual females. Electrophoresis 16:105-109.

19. King, M. 1993. Species Evolution: The Role of Chromosome Change. Cambridge University Press, Cambridge.

20. Mayr, E. 1969. Principles of Systematic Zoology. McGraw-Hill, New York.

21. Miller, L. I. 1983. Diversity of selected taxa of Globodera and Heterodera and their interspecific and intergeneric hybrids. Pages 207-220 in: Concepts in Nematode Systematics. The Systematic Association Special Volume 22. A. R. Stone, H. M. Platt, and L. F. Khalil, eds. Academic Press, London.

22. Mugniéry, D. 1979. Hybridization entre Globodera rostochiensis (Wollenweber) et G. pallida (Stone). Rev. Nematol. 2:153-159.

23. Pasteur, N., Pasteur, G., Bonhomme, F., Catalan, J., and Britton-Davidian, J. 1988. Practical Isozyme Genetics. John Wiley \& Sons, New York.

24. Perry, R. N., Plowright, R. A., and Webb, R. M. 1980. Mating between Pratylenchus penetrans and $P$. fallax in sterile culture. Nematologica 26: 125-129.

25. Pinkerton, J. N., Santo, G. S., and Mojtahedi, H. 1991. Population dynamics of Meloidogyne chitwoodi on Russet Burbank potatoes in relation to degree-day accumulation. J. Nematol. 23:283-290.

26. Potter, J. W., and Fox, J. A. 1965. Hybridization of Heterodera schachtii and $H$. glycines. Phytopathology 55:800-801.

27. Santos, S. M. N. De A. 1972. Production of male Meloidogyne spp. and attraction to their females. Nematologica 18:291-302.

28. Thiéry, M., Mugniéry, D., Fouville, D., and Schots, A. 1996. Hybridizations naturelle entre Globodera rostochiensis et G. pallida. Fundam. Appl. Nematol. 19:437-442.

29. Triantaphyllou, A. C. 1973. Environmental sex differentiation of nematodes in relation to pest management. Annu. Rev. Phytopathol. 11:441-462.

30. Triantaphyllou, A. C. 1985. Cytogenetics, cytotaxonomy and phylogeny of root-knot nematodes. Pages 113-126 in: An Advanced Treatise on Meloidogyne. Vol. 1, Biology and Control. J. N. Sasser and C. C. Carter, eds. North Carolina State University Graphics, Raleigh.

31. Triantaphyllou, A. C. 1985. Cytological methods for the study of oogenesis and reproduction of root-knot nematodes. Pages 107-114 in: An Advanced Treatise on Meloidogyne. Vol. 2, Methodology. K. R. Barker, C. C. Carter, and J. N. Sasser, eds. North Carolina State University Graphics, Raleigh.

32. Triantaphyllou, A. C. 1993. Hermaphroditism in Meloidogyne hapla. J. Nematol. 25:15-26.

33. Triantaphyllou, A. C., and Hirschmann, H. 1980. Cytogenetics and morphology in relation to evolution and speciation of plant-parasitic nematodes. Annu. Rev. Phytopathol. 18:333-359.

34. Van Meggelen, J. C., Karssen, G., Janssen, G. J. W., Verkerk-Bakker, B., and Janssen, R. 1994. A new race of Meloidogyne chitwoodi Golden et al, 1980? Fundam. Appl. Nematol. 17:93.

35. Wolf, H. G. 1987. Interspecific hybridization between Daphnia hyalina, D. galeata, and D. cucullata and seasonal abundances of these species and their hybrids. Hydrobiologia 145:213-217.

36. Yeates, G. W. 1970. Failure of Heterodera carotae, $H$. cruciferae and $H$. goettingiana to interbreed in vitro. Nematologica 16:153-154.

37. Zijlstra, C., Lever, A. E. M., Uenk, B. J., and Van Silfhout, C. H. 1995. Differences between ITS regions of isolates of root-knot nematodes $\mathrm{Mel}$ oidogyne hapla and M. chitwoodi. Phytopathology 85:1231-1237. 